EGU21-15068

https://doi.org/10.5194/egusphere-egu21-15068

EGU General Assembly 2021

(c) Author(s) 2021. This work is distributed under

the Creative Commons Attribution 4.0 License.

\title{
Novel UAV-based tools for assessing coastal grassland structure and function
}

\author{
Miguel Villoslada ${ }^{1}$, Thaisa Bergamo ${ }^{1,2}$, and Raymond Ward ${ }^{1,2}$ \\ ${ }^{1}$ Estonian University of Life Sciences, Institute of Agricultural and Environmental Sciences, Department of of Landscape \\ Management and Nature Protection, Tartu, Estonia (mpecina@emu.ee) \\ ${ }^{2}$ Centre for Aquatic Environments, School of the Environment and Technology, University of Brighton, Cockcroft Building, \\ Moulsecoomb, Brighton BN2 4GJ, United Kingdom
}

Coastal grasslands provide a wide range of ecosystem services worldwide. In order to better target conservation efforts in these ecosystems, it is necessary to develop highly accurate models that account for the spatial nature of ecosystem structure, processes and functions. Here, we present a novel set of UAV-based tools to accurately assess and map coastal grassland structure and functions. Firstly, a combination of UAV-derived datasets were used to produce vegetation indices and micro topographic models. A classification random forest algorithm was used to process the spectral and microtopography datasets and map the extent and spatial configuration of plant communities in coastal meadows in Estonia. The model accurately predicted the occurrence of plant communities with a very high kappa value.

In the second stage, a regression random forest algorithm was used to model and map aboveground biomass within the coastal grasslands sites. Subsequently, the above-ground biomass maps in combination with a mean-shift algorithm were used to assess grassland structural heterogeneity. The results were then related to management history at each study site, showing that continuous, monospecific grazing management tends to simplify grassland structure, which could in turn reduce the supply of a key regulation and maintenance ecosystem services: nursery and reproduction habitat for waders.

These results also indicate that UAV-based surveys can serve as reliable grassland monitoring tools and could aid in the development of site-specific management strategies. 